Punjab University Journal of Mathematics (2021),53(7),475-496

https://doi.org/10.52280/pujm.2021.530703

\title{
Corrections and Extensions in Left and Right Almost Semigroups
}

\author{
Nisar Ahmad, Syed Aleem Shah, Wali Khan Mashwani and Nasim Ullah \\ Institute of Numerical Sciences, \\ Kohat University of Science and Technology, Pakistan \\ Email: nisarnn22@yahoo.com
}

Received: 20 August, 2020 / Accepted: 07 June, 2021 / Published online: 27 July, 2021

\begin{abstract}
In this paper we elaborated the concept that on what conditions left almost semigroup (LA-Semigroup), right almost semigroup (RA-Semigroup) and a groupoid become commutative and further extended these results on medial, LA-Group and RA-Group. We proved that the relation of LA-Semigroup with left double displacement semigroup (LDD-semigroup), RA-Semigroup with left double displacement semigroup (RDD-semigroup) is only commutative property. We highlighted the errors in the recently developed results on LA-Semigroup and semigroup $[17,1,18]$ and proved that example discussed in [18] is semigroup with left identity but not paramedial. We extended results on locally associative LA-Semigroup explained in $[20,21]$ towards LA-Semigroup and RA-Semigroup with left zero and right zero respectively. We also discussed results on n-dimensional LA-Semigroup, n-dimensional RASemigroup, non commutative finite medials with three or more than three left or right identities and finite as well as infinite commutative idempotent medials not studied in literature.
\end{abstract}

\section{AMS (MOS) Subject Classification Codes: 13XX; 16XX; 17XX}

Key Words: LA-Semigroup; RA-Semigroup; Semigroup; Medial; Paramedial.

\section{INTRODUCTION}

Kazim and Naseeruddin [8] introduced the concept of LA-Semigroup, RA-Semigroup and Almost semigroup and elaborated that "If a groupoid $S$ satisfies the condition $(a b) c=$ $(c b) a$ then $S$ is LA-Semigroup and if groupoid $S$ holds condition $a(b c)=c(b a)$ then $S$ is RA-Semigroup and if $S$ satisfy both conditions then $S$ is almost semigroup. Mushtaq and Yousaf $[14,15]$ developed the idea of locally associative LA-Semigroups that hold property $(a a) a=a(a a)$ and elaborated that on what conditions LA-Semigroups becomes commutative group. In $[8,14,15]$ this is proved that "If $S$ is LA-Semigroup or RA-Semigroup then $S$ holds medial law or bisymmetry law i.e. $S$ satisfies the condition $(a b)(c d)=(a c)(b d)$ but the converse may not be true" and further this is also proved that the connection of 
LA-Semigroup and RA-Semigroup with semigroup is only commutative property. Later in 1984 Jazek and Kepka [7] developed results on almost semigroup. In [7, 16] LA-Semigroup is known as right modular groupoid, RA-Semigroup is known as left modular groupoid almost semigroup is known as bimodular groupoid. Seguier in 1904 [19] used the term semigroup for the first time in literature which is an algebraic structure $S$ that holds associative law i.e. $(a b) c=a(b c)$. Kimura and Yamada $[12,13]$ developed idea idempotent semigroup known as bands. Clifford, Preston and Howie $[3,6]$ wrote comprehensive books on semigroup theory and elaborated the concept of E-semigroups, regular semigroups, orthodox semigroups and also discussed about left ideals, right ideals, ideals and zero minimal ideals of semigroups. Kehayopulu [9] elaborated ordering of elements and constructed semigroups. Cho et al. [4] discussed some results on paramedial groupoid $S$ that satisfies condition $(a b)(c d)=(d b)(c a)$ and discussed its relation with commutative groupoid and medial. In $[11,10]$ concept of LA-Band also known as AG-Band has been developed and the relation of medial, paramedial and LA-Semigroup was also developed. Nisar et al. [2] introduced concept of LDD-Semigroup which is an algebraic structure $S$ that holds condition $(a b)(c d)=(c b)(a d)$ and explained that $R^{+}$is LDD-Semigroup w.r.t binary operations $a . b=e^{b}, a \eta b=b^{2}$. Some recent results were developed in LA-Semigroup, semigroup and paramedial groupoid in [20,21,1,17,18]. We have found error on semigroup in [1], error on LA-Semigroup in [17] and error on paramedial in [18]. Further following results were also proved:

Proved Results-1 in [8, 14, 16]:

If groupoid $S$ holds:

(a) left invertive law and associative law then $S$ is commutative semigroup as well as RASemigroup.

(b) right invertive law and associative law then $S$ is commutative semigroup as well as LASemigroup.

(c) associative law and commutative law then $S$ is LA-Semigroup as well as RA-Semigroup.

(d) left invertive law and commutative law then $S$ is commutative semigroup as well as RASemigroup.

(e) right invertive law and commutative law then $S$ is commutative semigroup as well as LA-Semigroup.

Proved Results-2 in $[8,14,4,2]$ :

If $S$ is:

(a) LA-Semigroup with right identity then $S$ is commutative monoid.

(b) RA-Semigroup with left identity then $S$ is commutative monoid.

(c) Medial with identity is commutative monoid.

(d) Paramedial with identity is commutative monoid.

(e) LDD-semigroup with right identity is commutative monoid.

(f) If $S$ is paramedial then $S$ is commutative: (i) If each element $\mathrm{x} \in S$ is idempotent (ii) $S$ contains identity element.

(g) If $S$ is LA-Monoid then $S$ is paramedial and by the converse paramedial with left identity is LA-Monoid. 


\section{NOTATIONS AND PRELIMINARIES}

Definition 2.1 : In literature a groupoid $\mathrm{S}$ is called left almost semigroup (LA-Semigroup) if for all elements $\mathrm{a}, \mathrm{b}, \mathrm{c} \in \mathrm{S}$ the left invertive law holds i.e. $(a b) c=(c b) a$.

Definition $2.2:$ In literature a groupoid $\mathrm{S}$ is called right almost semigroup (RA-Semigroup) if for all elements $\mathrm{a}, \mathrm{b}, \mathrm{c} \in \mathrm{S}$ right invertive law holds i.e. $a(b c)=c(b a)$.

Definition 2.3 : A groupoid $\mathrm{S}$ is medial if $\forall \mathrm{a}, \mathrm{b}, \mathrm{c}, \mathrm{d}$ lies in $\mathrm{S}, \mathrm{S}$ satisfies medial law or bisymmetry law i.e. $(a b)(c d)=(a c)(b d)$. Every LA-Semigroup and RA-Semigroup satisfies medial law but the converse may not be true.

Definition 2.4 : A groupoid $\mathrm{S}$ is semigroup if $\forall \mathrm{a}, \mathrm{b}, \mathrm{c} \in \mathrm{S}$ the condition $(a b) c=a(b c)$ holds.

Definition 2.5 : Semigroup $\mathrm{S}$ is called E-semigroup if the subset of S containing its idempotents also form semigroup.

Definition 2.6 : Semigroup $S$ is called regular semigroup if for each element $a \in S, \exists b \in$ S such that $a b a=a$ and $b a b=b$.

Definition 2.7 : Semigroup $\mathrm{S}$ is called orthodox semigroup if $\mathrm{S}$ is E-semigroup as well as regular semigroup.

Definition 2.8 : A semigroup $\mathrm{T}$ is called band if $\forall \mathrm{t} \in \mathrm{T}$ the condition $t^{2}=t$ is satisfied. Each band is orthodox semigroup.

Definition 2.9 : A groupoid $\mathrm{T}$ is called locally associative groupoid if $\forall \mathrm{t} \in \mathrm{T}$ condition $\left(t^{2}\right) t=t\left(t^{2}\right)$ is satisfied. If $\forall \mathrm{t} \in$ groupoid $\mathrm{T}$ the condition $t^{2}=t$ is satisfied then then $\mathrm{T}$ is locally associative groupoid. Every semigroup and commutative groupoid is locally associative but converse may not be true.

Definition 2.10 : LA-Semigroup $\mathrm{S}$ is called LA-Monoid if $\exists$ left identity $s_{i}$ in S such that for all $s_{j}$ in $\mathrm{S}$ the condition $s_{i} s_{j}=s_{j}$ holds.

Definition 2.11 : LA-Semigroup $\mathrm{S}$ is called LA-Group if there exists left identity $s_{i} \in \mathrm{S}$ and inverse of each element exists.

Definition 2.12 : RA-Semigroup $\mathrm{S}$ is called RA-Monoid if there exists right identity $s_{i} \in \mathrm{S}$ such that $\forall s_{j} \in \mathrm{S}, s_{j} s_{i}=s_{j}$.

Definition 2.13 : RA-Semigroup $\mathrm{S}$ is called RA-Group if $\exists$ right identity element $s_{i} \in \mathrm{S}$ and inverse of each element exists.

Definition 2.14 : A groupoid $\mathrm{S}$ is called paramedial if $\forall \mathrm{a}, \mathrm{b}, \mathrm{c}, \mathrm{d} \in \mathrm{S}$ the condition $(a b)(c d)=(d b)(c a)$ holds.

Definition 2.15 : Zero semigroup $S$ is such semigroup that contains zero element a and $\forall$ $\mathrm{b}, \mathrm{c} \in \mathrm{S}$ the condition $b b=c c=b c=c b=a$ is satisfied. Zero semigroup is also called zeropotent groupoid and this is such commutative semigroup which is not regular.

Definition 2.16: A groupoid L is called left double displacement semigroup (LDD-semigroup) if $\forall \mathrm{a}, \mathrm{b}, \mathrm{c}, \mathrm{d} \in \mathrm{L}$ the condition $(a b)(c d)=(c b)(a d)$ ".

Definition 2.17 : A groupoid $\mathrm{M}$ is called right double displacement semigroup (RDDsemigroup) if $\forall \mathrm{a}, \mathrm{b}, \mathrm{c}, \mathrm{d} \in \mathrm{M}$ the condition $(a b)(c d)=(a d)(c b)$ ".

This property $(a b)(c d)=(c b)(a d)$ is left double displacement law (LDD-law) and $(a b)(c d)=$ $(a d)(c b)$ is right double displacement law (RDD-law).

Throughout this paper we will use following notations: $R$ for set of real numbers. 
$Q$ for set of rational numbers.

$Z$ for set of integer.

$k$ for finite positive integer.

$Z_{n}$ for $\bmod n$.

$S$ for LA-Semigroup.

$T$ for RA-Semigroup.

$X$ for groupoid.

$G$ for commutative group.

$(G,$.$) for commutative group.$

$H$ for group.

$U$ and $V$ for medial.

$Y$ for almost semigroup.

$D$ for semigroup.

$f$ and $g$ for functions defined from $R \rightarrow R, Q \rightarrow Q, Z \rightarrow Z$ and $Z_{n} \rightarrow Z_{n}$.

\section{Main Results}

We divided this section into four subsections. Subsection 3.1 deals with the results on LA-Semigroup and RA-Semigroups. In subsection 3.2 we highlighted errors in recently developed results on LA-Semigroup and semigroup in $[1,17]$ and extended results on locally associative LA-Semigroups in [20,21]. In subsection 3.3 we highlighted that example discussed in [18] is semigroup with left identity but not paramedial. In subsection 3.4 we developed some results on n-dimensional LA-Semigroup as well as n-dimensional RASemigroup, non commutative medials and commutative medials.

\subsection{Results on LA-Semigroup and RA-Semigroup.}

In this section we discussed relation of LA-Semigroup and RA-Semigroups with commutative semigroup. We also developed the concept that how medial can be constructed from commutative group. Further we elaborated the relation of medial, LA-Semigroup and RA-Semigroup with LDD-Semigroup, RDD-Semigroup and paramedial groupoid by the following results:

Theorem 3.2. If LA-Semigroup $S$ holds the condition $(a b) c=(a c) b \forall a, b, c \in S$, then $S$ is commutative.

Proof. From the given conditions we do the following steps:

$(a b)(c d)=((c d) b) a=((c b) d) a$

$$
\begin{aligned}
& =((c b) a) d=((a b) c) d \\
& =((a b) d) c=(c d)(a b)
\end{aligned}
$$

This shows that $S$ is commutative LA-Semigroup and every commutative LA-Semigroup is commutative semigroup as well as commutative RA-Semigroup proved in [8, 14].

Remark 3.3. $S$ is is commutative if $\forall a, b, c, d \in S, S$ holds any of the following conditions:
(i) $(a b) c=(b a) c$
(ii) $(a b) c=(b c) a$ or $(a b) c=(c a) b$
(iii) $a(b c)=(c b) a$ 
Theorem 3.4. If a groupoid $X$ satisfies the conditions $(a b) c=(b a) c$ and $(a b) c=(a c) b \forall$ $a, b, c \in X$ then $S$ is commutative semigroup.

Proof. Using the given conditions we do the following steps: $(a b) c=(a c) b=(c a) b=(c b) a$ which shows that $S$ is LA-Semigroup.

$(c b) a=(b c) a=(a c) b$ which shows that $S$ satisfies the conditions $(a b) c=(a c) b$.

So by using theorem-3.2 this is straightforward that $X$ is commutative semigroup.

Theorem 3.5. If $X$ satisfies conditions $(a b) c=(b a) c$ and $(a b) c=(b c) a \forall a, b, c \in X$ then $X$ is commutative semigroup.

Proof. From the given conditions we do the following steps:

$(a b) c=(b c) a=(c b) a$ which shows that $S$ is LA-Semigroup.

So $(a b) c=(b a) c=(c a) b=(a c) b$.

Using theorem 3.3 this is quite easy to prove that $X$ is commutative semigroup.

Theorem 3.6. If $X$ holds conditions $a(b c)=a(c b)$ and $a(b c)=b(a c) \forall a, b, c \in$, then $X$ is commutative semigroup.

Proof. From the given conditions we have $a(b c)=a(c b)=c(a b)=c(b a)$ so $S$ is RASemigroup.

To prove that $S$ is commutative we do the following steps:

$(a b)(c d)=d(c(a b))=d(a(c b))$

$$
\begin{aligned}
& =a(d(c b)=a(b(c d)) \\
& =b(a(c d))=(c d)(a b)
\end{aligned}
$$

Thus $X$ is commutative.

So $a(b c)=a(c b)=c(a b)=(a b) c$ which shows that $X$ is semigroup.

Remark 3.7. RA-Semigroup $T$ is commutative if $\forall a, b, c \in T, T$ holds any condition from the following
(i) $a(b c)=a(c b)$
(ii) $a(b c)=b(a c)$
(iii) $(a b) c=c(b a)$
(iii) $a(b c)=b(c a)$ or $a(b c)=b(c a)$

Theorem 3.8. If $T$ satisfies condition $a(b c)=(b a) c \forall a, b, c \in T$, then $T$ is commutative.

Proof. Using the given conditions we have $a(b c)=c(b a)=(b c) a=(b a) c$ so we do the following steps:

$$
\begin{gathered}
(a b)(c d)=d(c(a b))=d((a b) c) \\
=d(b(a c))=(b d)(a c)
\end{gathered}
$$

Every RA-Semigroup satisfies medial law proved in $[8,14]$.

So $(a b)(c d)=(a c)(b d)=(c d)(a b) \Rightarrow T$ is commutative.

Remark 3.9. If we define binary operation on finite groupoid $X$ by $a b=V(c)$ where $V(c)$ means value of the Cth entry and $C=b^{\text {th }}$ entry $-a^{\text {th }}$ entry $+i^{\text {th }}$ entry under the mod $m$ then following conditions hold:
(a) $i^{\text {th }}$ element is left identity.
(b) S holds left invertive law.
(c) If order of $S$ i.e $n(S)$ is even then the subset of $S$ say $S_{1}=\left\{s_{i}, s_{j}\right\}$ forms cyclic group and $|i-j|=|j-i|=m / 2$.
(d) If order of $S$ i.e $n(S)$ is odd then subset of $S$ say $S_{I}$ that contains only left identity 
element $s_{i}$ forms groupoid and LA-Semigroup which is trivial.

(e) Every element $s_{j}$ is self inverse i.e. $s_{j} s_{j}=s_{i}$.

Example: If binary operation $*$ is defined on $A_{100}=\left\{a_{1}, a_{2}, a_{3}, a_{4}, \ldots, a_{100}\right\}$ in such way that $a_{23}$ is idempotent element and column entries follow one element preceding difference pattern and the row entries follow the one element succeeding difference pattern then $a_{23}$ is left identity and the subset of $A_{100}$ say $A_{100 C}=\left\{a_{23}, a_{73}\right\}$ forms cyclic group and this is quite clear that $|73-23|=|23-73|=50=100 / 2$.

Remark 3.10. If we define binary operation on finite groupoid $X$ by $a b=V(c)$ where $V(c)$ means value of the Cth entry and $C=a^{\text {th }}$ entry $-b^{\text {th }}$ entry $+i^{\text {th }}$ entry under the mod $m$ then following conditions hold:

(a) $i^{\text {th }}$ element is right identity.

(b) S holds right invertive law.

(c) If order of $S$ i.e $n(S)$ is even then the subset of $S$ say $S_{1}=\left\{s_{i}, s_{j}\right\}$ forms cyclic group and $|i-j|=|j-i|=m / 2$.

(d) If order of $S$ i.e $n(S)$ is odd then subset of $S$ say $S_{I}$ that contains only right identity element $s_{i}$ forms groupoid and RA-Semigroup which is trivial.

(e) Every element $s_{j}$ is self inverse i.e. $s_{j} s_{j}=s_{i}$.

Example: If binary operation on $B_{4}=\left\{b_{1}, b_{2}, b_{3}, \ldots \ldots . b_{488}\right\}$ is defined in such way that $b_{175}$ is idempotent element and all elements follow one element succeeding difference pattern in column and one element preceding element pattern in row then the subset of $B_{488}$ that contains elements $b_{175}$ and $b_{419}$ forms cyclic group and clearly $|419-175|=$ $|175-419|=244=488 / 2$.

\section{Remarks On LA-Group:}

(a) In case of odd number of elements only left identity element $s_{i}$ is both (left and right) regular.

(b) In case of even number of elements only two elements left identity element $s_{i}$ and the element $s_{j}$ that forms cyclic group are both left and right regular.

(c) In case of even number of elements every element other than left identity $s_{i}$ and $s_{j}$ that forms cyclic group is right regular w.r.t itself i.e. $a^{2} a=a$ and for each a, $\exists \mathrm{b}$ such that $b a^{2}=a$ i.e. each a other than $s_{i}$ and $s_{j}$ is left regular with other element $\mathrm{b}$. When number of elements are odd then also this condition holds but in this case only $s_{i}$ is both (left and right) regular with itself.

(d) When $s_{i}$ is left identity then the elements a and $\mathrm{b}$ that satisfies the condition $b a^{2}=a$ and $a b^{2}=b$ also satisfies the condition that $a_{*} s_{i}=b$ and $b_{*} s_{i}=a$.

\section{Remarks On RA-Group:}

(a) In case of odd number of elements only right identity element $s_{i}$ is both (left and right) regular.

(b) In Case of even number of elements only two elements right identity element $s_{i}$ and the element $s_{j}$ that forms cyclic group are both left and right regular.

(c) In Case of even number of elements every element other than right identity $s_{i}$ and $s_{j}$ that forms cyclic group is left regular w.r.t itself i.e. $a a^{2}=a$ and for each $\mathrm{a}, \exists \mathrm{b}$ such that $a^{2} b=a$ i.e. each a other than $s_{i}$ and $s_{j}$ is right regular with other element $\mathrm{b}$. When number 
of elements are odd then also this condition holds but in this case only $s_{i}$ is both (left and right) regular with itself.

(d) When $s_{i}$ is right identity then the elements a and $\mathrm{b}$ that satisfies the condition $a^{2} b=a$ and $b^{2} a=b$ also satisfies the condition that $* s_{i} a=b$ and $* s_{i} b=a$.

Corollary 3.11. If $(G,$.$) is commutative group and we define binary operation \mu$ on $G$ by $a \mu b=b^{k} \cdot a^{-1}$ where $k$ is non negative finite integer then $G$ satisfies medial law.

Corollary 3.12. If $(G,$.$) is commutative group and we define binary operation \nu$ on $G$ by $a \nu b=a^{k} \cdot b^{-1}$ and $k$ is finite positive integer then $G$ satisfies medial law.

Note in Corollary 3.11 and 3.12: If $k=1$ then $(G, \mu)$ is LA-Group in which each element is self inverse and $(G, \nu)$ is RA-Group in which each element is self inverse respectively.

Theorem 3.13. If $S$ is locally associative LA-Group in which each element is self inverse then $S$ is commutative group.

Proof. Let e be the left identity in LA-Group $S$ then by using the given conditions, we prove theorem by following steps:

$(a a) a=e a=a=a(a a)=a e$

Thus $\forall \mathrm{a} \in S$ the condition $e a=a e=a$ is satisfied which shows e is also right identity in $S$.

So using "Proved Results 1", $S$ is commutative group.

Theorem 3.14. If T is locally associative RA-Group in which each element is self inverse then $T$ is commutative group.

Proof. Let e be the right identity in RA-Group $T$ then to prove the theorem we do the following steps:

$(a a) a=e a=a=a(a a)=a e$

Thus $\forall \mathrm{a} \in T$ the condition $e a=a e=a$ is satisfied which shows that e is also left identity in $T$.

So using "Proved Results 1", $T$ is commutative group.

Theorem 3.15. If $S$ satisfies the condition $(a b)(c d)=((b a) c) d \forall a, b, c, d \in S$ or $T$ satisfies condition $(u v)(w x)=u(v(x w)) \forall a, b, c, d \in T$ then $S$ and $T$ are paramedial.

Proof. Using the given conditions we prove results by the following steps:

$(a b)(c d)=((b a) c) d=(d c)(b a)=(d b)(c a) \Rightarrow S$ is paramedial.

$(u v)(w x)=u(v(x w))=(x w)(v u)=(x v)(w v) \Rightarrow T$ is paramedial.

Theorem 3.16. If $Y$ is almost semigroup and paramedial then $Y$ is commutative semigroup.

Proof. From the given conditions $\forall \mathrm{a}, \mathrm{b}, \mathrm{c}, \mathrm{d} \in Y$ we have $(a b)(c d)=(a c)(b d)=$ $(d b)(c a)=(d c)(b a)$ and also $(a b) c=(c b) a$ and $a(b c)=c(b a)$.

There is no direct way to prove this so we take five elements $a, b, c, d$ and $f$ and prove the theorem by the following steps: 


$$
\begin{aligned}
(a b)((c d) f) & =(a b)((f d) c)=c((f d)(a b)) \\
= & c((b d))(a f))=c((b a)(d f)) \\
= & (d f)((b a) c)=(d f)((c a) b) \\
= & b((c a)(d f))=b((c d)(a f)) \\
= & (a f)((c d) b) .
\end{aligned}
$$

From the steps this is clear that $\forall \mathrm{a}, \mathrm{b}, \mathrm{x}$ and $\mathrm{f} \in Y$ the condition $(a b)(x f)=(a f)(x b)$ holds.

So this shows that $Y$ is also RDD-semigroup. So using medial, paramedial and RDD laws we prove the theorem by following steps:

$$
\begin{aligned}
(a b)(c d) & =(a c)(b d)=(d c)(b a) \\
& =(b c)(d a)=(b d)(c a) \\
& =(a d)(c b)=(c d)(a b)
\end{aligned}
$$

Lemma 3.17. If $U$ is medial and LDD-Semigroup then $\forall a, b, c, d \in U, U$ satisfies the following conditions hold:
(i) $(a b)(c d)=(b a)(c d)$
(ii) $(a b)(c d)=(c a)(b d)$
(iii) $(a b)(c d)=(b c)(a d)$
(iv) $(a b) c=(b a) c$

Theorem 3.18. If $S$ is LA-Semigroup as well as LDD-semigroup then $S$ is commutative.

Proof. Using lemma 3.17 and theorems 3.2 and 3.3 this is straightforward.

Lemma 3.19. If $U$ is medial and RDD-Semigroup then $\forall a, b, c, d \in U, U$ satisfies the following conditions hold:
(i) $(a b)(c d)=(a b)(d c)$
(ii) $(a b)(c d)=(a c)(d b)$
(iii) $(a b)(c d)=(a d)(c b)$
(iv) $a(b c)=a(c b)$

Theorem 3.20. If $T$ is RA-Semigroup as well as RDD-semigroup then $T$ is commutative.

Proof. Using lemma 3.19, theorems 3.7 and theorem 3.8 the proof is straightforward.

Remark 3.21. We investigated following results on almost semigroups:

(i) Almost semigroup $Y$ is commutative if $Y$ is:

$\begin{array}{llll}\text { (a) Paramedial } & \text { (b) Semigroup } & \text { (c)LDD-semigroup } & \text { (d) RDD-semigroup }\end{array}$

(ii) The proof of following theorems are not easy by direct way:

(a) If $S$ is LA-Semigroup and LDD-Semigroup then $S$ is commutative.

(b) If $T$ is RA-Semigroup and RDD-Semigroup then $T$ is commutative.

(c) If almost semigroup $Y$ is paramedial then $Y$ is commutative.

We have to use lemmas and results on the relation of medial with LDD-Semigroup or RDDSemigroup to prove (a) or (b) respectively, and use the idea of taking five or six elements to prove (c).

\subsection{Corrections and Extensions.}

In this section we highlighted errors in $[1,17]$ explained by the following tables and discussion:

Indicating Errors in LA-Semigroup and Semigroup Results: 
Imtiaz et al. [1] took set $A=\{p, q, r, s, t, u\}$ constructed example 3.24 on semigroup and Noor et al. [17] took set $B=\{a, b, c\}$ and constructed example on LA-Semigroup by the following tables:

TABle 1. Table For $A$ and $B$

\begin{tabular}{|c|c|c|c|c|c|c|c|c|c|c|c|}
\hline$*$ & $\mathrm{p}$ & $\mathrm{q}$ & $\mathrm{r}$ & $\mathrm{s}$ & $\mathrm{t}$ & $\mathrm{u}$ & & $*$ & $\mathrm{a}$ & $\mathrm{b}$ & $\mathrm{c}$ \\
\hline $\mathrm{p}$ & $\mathrm{q}$ & $\mathrm{s}$ & $\mathrm{q}$ & $\mathrm{q}$ & $\mathrm{u}$ & $\mathrm{q}$ & & $\mathrm{a}$ & $\mathrm{a}$ & $\mathrm{a}$ & $\mathrm{a}$ \\
\hline $\mathrm{q}$ & $\mathrm{t}$ & $\mathrm{q}$ & $\mathrm{q}$ & $\mathrm{q}$ & $\mathrm{q}$ & $\mathrm{q}$ & & $\mathrm{b}$ & $\mathrm{c}$ & $\mathrm{c}$ & $\mathrm{c}$ \\
\hline $\mathrm{r}$ & $\mathrm{q}$ & $\mathrm{q}$ & $\mathrm{q}$ & $\mathrm{q}$ & $\mathrm{q}$ & $\mathrm{q}$ & & $\mathrm{c}$ & $\mathrm{a}$ & $\mathrm{a}$ & $\mathrm{c}$ \\
\hline $\mathrm{s}$ & $\mathrm{u}$ & $\mathrm{q}$ & $\mathrm{q}$ & $\mathrm{q}$ & $\mathrm{q}$ & $\mathrm{q}$ & & & & & \\
\hline $\mathrm{t}$ & $\mathrm{q}$ & $\mathrm{q}$ & $\mathrm{q}$ & $\mathrm{q}$ & $\mathrm{q}$ & $\mathrm{q}$ & & & & & \\
\hline $\mathrm{u}$ & $\mathrm{q}$ & $\mathrm{q}$ & $\mathrm{q}$ & $\mathrm{q}$ & $\mathrm{q}$ & $\mathrm{q}$ & & & & & \\
\hline
\end{tabular}

Clearly $A$ is not semigroup because $(p t) t=u t=q$ but $p(t t)=p q=s$ and also $B$ is not LA-Semigroup because $(b b) c=c c=c$ but $(c b) b=a b=a$. So the examples in $[1,17]$ are not correct.

Further we have some LA-Semigroup tables which are for set $S=\{a, b, c\}$.

TABLE 2. Tables For $\mathrm{S}$

\begin{tabular}{|c|c|c|c|c|c|c|c|c|c|c|c|c|c|c|c|c|c|c|}
\hline$*$ & $\mathrm{a}$ & $\mathrm{b}$ & $\mathrm{c}$ & &. & $\mathrm{a}$ & $\mathrm{b}$ & $\mathrm{c}$ & & $\mu$ & $\mathrm{a}$ & $\mathrm{b}$ & $\mathrm{c}$ & & $\nu$ & $\mathrm{a}$ & $\mathrm{b}$ & $\mathrm{c}$ \\
\hline $\mathrm{a}$ & $\mathrm{a}$ & $\mathrm{b}$ & $\mathrm{c}$ & & $\mathrm{a}$ & $\mathrm{a}$ & $\mathrm{a}$ & $\mathrm{a}$ & & $\mathrm{a}$ & $\mathrm{c}$ & $\mathrm{c}$ & $\mathrm{b}$ & & $\mathrm{a}$ & $\mathrm{c}$ & $\mathrm{c}$ & $\mathrm{c}$ \\
\hline $\mathrm{b}$ & $\mathrm{c}$ & $\mathrm{a}$ & $\mathrm{b}$ & & $\mathrm{b}$ & $\mathrm{a}$ & $\mathrm{a}$ & $\mathrm{a}$ & & $\mathrm{b}$ & $\mathrm{b}$ & $\mathrm{b}$ & $\mathrm{b}$ & & $\mathrm{b}$ & $\mathrm{b}$ & $\mathrm{b}$ & $\mathrm{b}$ \\
\hline $\mathrm{c}$ & $\mathrm{b}$ & $\mathrm{c}$ & $\mathrm{a}$ & & $\mathrm{c}$ & $\mathrm{a}$ & $\mathrm{b}$ & $\mathrm{a}$ & & $\mathrm{c}$ & $\mathrm{b}$ & $\mathrm{b}$ & $\mathrm{b}$ & $\mathrm{c}$ & $\mathrm{b}$ & $\mathrm{b}$ & $\mathrm{b}$ \\
\hline & & & & & & & & & & & & & & & & & & \\
\hline$\eta$ & $\mathrm{a}$ & $\mathrm{b}$ & $\mathrm{c}$ & & $\rho$ & $\mathrm{a}$ & $\mathrm{b}$ & $\mathrm{c}$ & & $\phi$ & $\mathrm{a}$ & $\mathrm{b}$ & $\mathrm{c}$ & & $\chi$ & $\mathrm{a}$ & $\mathrm{b}$ & $\mathrm{c}$ \\
\hline $\mathrm{a}$ & $\mathrm{a}$ & $\mathrm{a}$ & $\mathrm{a}$ & $\mathrm{a}$ & $\mathrm{a}$ & $\mathrm{a}$ & $\mathrm{a}$ & & $\mathrm{a}$ & $\mathrm{a}$ & $\mathrm{a}$ & $\mathrm{a}$ & & $\mathrm{a}$ & $\mathrm{b}$ & $\mathrm{b}$ & $\mathrm{b}$ \\
\hline $\mathrm{b}$ & $\mathrm{a}$ & $\mathrm{a}$ & $\mathrm{a}$ & $\mathrm{b}$ & $\mathrm{a}$ & $\mathrm{a}$ & $\mathrm{a}$ & & $\mathrm{b}$ & $\mathrm{c}$ & $\mathrm{a}$ & $\mathrm{a}$ & & $\mathrm{b}$ & $\mathrm{b}$ & $\mathrm{b}$ & $\mathrm{b}$ \\
\hline $\mathrm{c}$ & $\mathrm{b}$ & $\mathrm{a}$ & $\mathrm{b}$ & $\mathrm{c}$ & $\mathrm{a}$ & $\mathrm{c}$ & $\mathrm{a}$ & & $\mathrm{c}$ & $\mathrm{a}$ & $\mathrm{a}$ & $\mathrm{a}$ & $\mathrm{c}$ & $\mathrm{b}$ & $\mathrm{a}$ & $\mathrm{b}$ \\
\hline
\end{tabular}

There are also some other ways to construct LA-Semigroups of order three which are explained in [21]. Here we only mentioned some of them. The concept that every finite LA-Semigroup can be converted into RA-Semigroup by just taking the transpose of the LA-matrix generated by the binary operation applied on elements in some finite groupoid may not be correct e.g. if we have set $\bar{A}$ with the following tables:

TABLE 3. Table for LA-Semigroup not Converted to RA-Semigroup

\begin{tabular}{|c|c|c|c|c|c|c|c|c|c|c|}
\hline$*$ & $\mathrm{a}$ & $\mathrm{b}$ & $\mathrm{c}$ & $\mathrm{d}$ & &. & $\mathrm{a}$ & $\mathrm{b}$ & $\mathrm{c}$ & $\mathrm{d}$ \\
\hline $\mathrm{a}$ & $\mathrm{a}$ & $\mathrm{a}$ & $\mathrm{a}$ & $\mathrm{a}$ & & $\mathrm{a}$ & $\mathrm{a}$ & $\mathrm{a}$ & $\mathrm{a}$ & $\mathrm{a}$ \\
\hline $\mathrm{b}$ & $\mathrm{a}$ & $\mathrm{a}$ & $\mathrm{a}$ & $\mathrm{a}$ & & $\mathrm{b}$ & $\mathrm{a}$ & $\mathrm{a}$ & $\mathrm{a}$ & $\mathrm{a}$ \\
\hline $\mathrm{c}$ & $\mathrm{a}$ & $\mathrm{a}$ & $\mathrm{a}$ & $\mathrm{a}$ & & $\mathrm{c}$ & $\mathrm{a}$ & $\mathrm{a}$ & $\mathrm{a}$ & $\mathrm{d}$ \\
\hline $\mathrm{d}$ & $\mathrm{a}$ & $\mathrm{a}$ & $\mathrm{d}$ & $\mathrm{a}$ & & $\mathrm{d}$ & $\mathrm{a}$ & $\mathrm{a}$ & $\mathrm{a}$ & $\mathrm{a}$ \\
\hline
\end{tabular}


In both cases $\bar{A}$ is LA-Semigroup but not RA-Semigroup, so the concept that each finite LA-Semigroup can be converted into RA-Semigroup by taking the transpose in the table of some LA-Semigroup structure is not correct.

We investigated that in most cases by changing the order of elements in the table usually by taking the transpose some LA-matrix table, we get RA-matrix table.

Further we constructed finite LA-Semigroups of order 4 without any idempotent element by the following tables:

TABLE 4. Table for LA-Semigroup without Idempotent

\begin{tabular}{|c|c|c|c|c|c|c|c|c|c|c|}
\hline$*$ & $\mathrm{a}$ & $\mathrm{b}$ & $\mathrm{c}$ & $\mathrm{d}$ & & $\mathrm{c}$ & $\mathrm{a}$ & $\mathrm{b}$ & $\mathrm{c}$ & $\mathrm{d}$ \\
\hline $\mathrm{a}$ & $\mathrm{b}$ & $\mathrm{c}$ & $\mathrm{a}$ & $\mathrm{d}$ & & $\mathrm{a}$ & $\mathrm{b}$ & $\mathrm{d}$ & $\mathrm{c}$ & $\mathrm{a}$ \\
\hline $\mathrm{b}$ & $\mathrm{d}$ & $\mathrm{a}$ & $\mathrm{c}$ & $\mathrm{b}$ & & $\mathrm{b}$ & $\mathrm{c}$ & $\mathrm{a}$ & $\mathrm{b}$ & $\mathrm{d}$ \\
\hline $\mathrm{c}$ & $\mathrm{c}$ & $\mathrm{b}$ & $\mathrm{d}$ & $\mathrm{a}$ & & $\mathrm{c}$ & $\mathrm{a}$ & $\mathrm{c}$ & $\mathrm{d}$ & $\mathrm{b}$ \\
\hline $\mathrm{d}$ & $\mathrm{a}$ & $\mathrm{d}$ & $\mathrm{b}$ & $\mathrm{c}$ & & $\mathrm{d}$ & $\mathrm{d}$ & $\mathrm{b}$ & $\mathrm{a}$ & $\mathrm{c}$ \\
\hline
\end{tabular}

Extensions in Locally Associative LA-Semigroup Discussed in [20, 21] Shah et al. [20, 21] constructed new example on locally associative LA-Semigroup with zero element in which they took set $S=\{0,1,2\}$ and binary operation. was defined in such way in the table $1.0=0$ and all other entries were substituted by 2 , so 2 is zero element of the structure $(S,$.$) . Also we are using the concept of zero semigroup A in which binary$ operation is defined in such way that any two elements are operated and we get zero element of that structure A or in simple words a semigroup in which the we get only zero element in the cayley table. But in Case- 3 we do some changes and there are two elements $a_{1}$ and $a_{2}$ which are not zero elements but they disturbs the commutative and associative properties of the whole structure in which either $a_{1} a_{2}=a_{2}$ or $a_{1} a_{2}=a_{1}$ or we have the other situation in which $a_{2} a_{1}=a_{1}$ or $a_{2} a_{1}=a_{2}$. We extend this concept to such LA-Semigroup C in such way that we can have two, three, four or more idempotents in which one idempotent is the zero element of the LA-Semigroup $\mathrm{C}$ and the subset $C_{1}$ of $\mathrm{C}$ having these idempotent members of $\mathrm{C}$ forms commutative orthodox semigroup and $C_{1}$ is also the ideal of $\mathrm{C}$. We take $C_{4}=\left\{c_{1}, c_{2}, c_{3}, c_{4}\right\}, C_{5}=\left\{c_{1}, c_{2}, c_{3}, c_{4}, c_{5}\right\}$ and $C_{6}=\left\{c_{1}, c_{2}, c_{3}, c_{4}, c_{5}, c_{6}\right\}$ and binary operation $\eta$ (eta) is defined on $C_{1}, C_{2}$ and $C_{3}$ elaborated in "Table For $C_{4}, C_{5}$ and $C_{6}{ }^{\prime}$.

TABle 5. Table For $C_{4}, C_{5}$ and $C_{6}$

\begin{tabular}{|l|l|l|l|l|l|l|l|l|l|l|l|l|l|l|l|l|l|l|l|}
\hline & $c_{1}$ & $c_{2}$ & $c_{3}$ & $c_{4}$ & & & $c_{1}$ & $c_{2}$ & $c_{3}$ & $c_{4}$ & $c_{5}$ & & & $c_{1}$ & $c_{2}$ & $c_{3}$ & $c_{4}$ & $c_{5}$ & $c_{6}$ \\
\hline$c_{1}$ & $c_{1}$ & $c_{1}$ & $c_{1}$ & $c_{1}$ & & $c_{1}$ & $c_{1}$ & $c_{1}$ & $c_{1}$ & $c_{1}$ & $c_{1}$ & $c_{1}$ & $c_{1}$ & $c_{1}$ & $c_{1}$ & $c_{1}$ & $c_{1}$ & $c_{1}$ \\
\hline$c_{2}$ & $c_{1}$ & $c_{1}$ & $c_{1}$ & $c_{1}$ & & $c_{2}$ & $c_{1}$ & $c_{1}$ & $c_{1}$ & $c_{1}$ & $c_{1}$ & $c_{2}$ & $c_{1}$ & $c_{1}$ & $c_{1}$ & $c_{1}$ & $c_{1}$ & $c_{1}$ \\
\hline$c_{3}$ & $c_{1}$ & $c_{2}$ & $c_{1}$ & $c_{1}$ & & $c_{3}$ & $c_{1}$ & $c_{2}$ & $c_{1}$ & $c_{1}$ & $c_{1}$ & $c_{3}$ & $c_{1}$ & $c_{2}$ & $c_{1}$ & $c_{1}$ & $c_{1}$ & $c_{1}$ \\
\hline$c_{4}$ & $c_{1}$ & $c_{1}$ & $c_{1}$ & $c_{4}$ & & $c_{4}$ & $c_{1}$ & $c_{1}$ & $c_{1}$ & $c_{4}$ & $c_{1}$ & $c_{4}$ & $c_{1}$ & $c_{1}$ & $c_{1}$ & $c_{4}$ & $c_{1}$ & $c_{1}$ \\
\hline & & & & & & $c_{5}$ & $c_{1}$ & $c_{1}$ & $c_{1}$ & $c_{1}$ & $c_{5}$ & & $c_{5}$ & $c_{1}$ & $c_{1}$ & $c_{1}$ & $c_{1}$ & $c_{5}$ & $c_{1}$ \\
\hline & & & & & & & & & & & & & $c_{6}$ & $c_{1}$ & $c_{1}$ & $c_{1}$ & $c_{1}$ & $c_{1}$ & $c_{6}$ \\
\hline
\end{tabular}




\section{Scheme In Locally Associative LA-Semigroups with Zero Element:}

Select two elements which are neither left nor right zero and also not idempotents say $c_{1}$ and $c_{2}$ and use this condition that either $c_{1} c_{2}=c_{2}$ and $c_{2} c_{1}=c_{o}$ i.e. zero element or $c_{1} c_{2}=c_{1}$ and $c_{2} c_{1}=c_{o}$. This clearly makes the groupoid neither commutative nor associative but this groupoid will be locally associative LA-Semigroup.

Clearly from the tables the groupoids $C_{4}, C_{5}$ and $C_{6}$ are neither commutative nor associative however they are locally associative LA-Semigroups with zero element $c_{1}$. The subset of $C_{4}$ say $C_{4 A}=\left\{c_{1}, c_{4}\right\}$ is commutative orthodox semigroup and ideal of $C_{4}$. The set $C_{5 A}=\left\{c_{1}, c_{4}, c_{5}\right\}$ is commutative orthodox semigroup and ideal of $C_{5}$. Similarly this is also clear from the table that $C_{6 A}=\left\{c_{1}, c_{4}, c_{5}, c_{6}\right\}$ is commutative orthodox semigroup and ideal of $C_{6}$.

\section{Remarks in Locally Associative LA-Semigroup with Zero Element:}

a. This is locally associative LA-Semigroup with zero element.

b. Zero element with other idempotent(s) forms commutative orthodox semigroup as well as ideal of LA-Semigroup C.

c. Zero element with two non idempotent members that violates commutative and associative property only form LA-Semigroup.

d. Each commutative orthodox semigroup which is also ideal of LA-Semigroup structure must contain zero element.

e. The product of two ideals $C_{I}$ and $C_{J}$ is $\{0\}$ when $C_{I}=\left\{0, c_{i}^{1}, c_{i}^{2}, c_{i}^{3}, \ldots, c_{i}^{n}\right\}$ and $C_{J}=$ $\left\{0, c_{j}^{1}, c_{j}^{2}, c_{j}^{3}, \ldots, c_{j}^{n}\right\}$ where $I=\left\{c_{i}^{1}, c_{i}^{2}, c_{i}^{3}, \ldots, c_{i}^{n}\right\}$ and $J=\left\{c_{j}^{1}, c_{j}^{2}, c_{j}^{3}, \ldots, c_{j}^{n}\right\}$ are disjoint sets. Here we are not taking power of elements, here this means $i_{1}, i_{2}, \ldots, i_{n}$ and $j_{1}, j_{2}$, $\ldots ., j_{n}$. We can simply write $C_{I}=\left\{0, i_{1}, i_{2}, i_{3}, \ldots ., i_{n}\right\}$ and $C_{J}=\left\{0, j_{1}, j_{2}, j_{3}, \ldots ., j_{n}\right\}$.

f. LA-Semigroup $\mathrm{C}$ has many proper ideals but this has no zero minimal ideal and the trivial zero minimal ideal is $\{0\}$.

g. This is the case of LA-Semigroup which is the not regular, neither left not right regular. h. The idempotents of this locally associative LA-Semigroup forms regular as well as inverse semigroup because all the idempotent elements commute with each other.

Practical Application: A useful practical application can be for setting some password for some bank locker or some safe if by clicking any two digits entries give specific same number but two entries give different number e.g. if password is to close and open some safe or vault is 55557 then $1.1,2.1,3.1$ and 4.1 execute 5 but 6.7 executes 7 and 7.6 executes 5 and the lock can be closed or opened.

For securing the important documents in some safe or vault the pattern discussed in $[20,21]$ and in it's extensions can be useful tool. Also from 0 to9 we can select two or more than idempotents digits and password can be set for securing digital locks of safes or vaults. This can be used in cryptography procedures. The more complicated will be the binary operation the more safe and secure will be information.

Extension-1 in [21]: We extended this idea of LA-Semigroup in which non cyclic but commutative group (Klein-4 group) is contained if we just have set $C_{7}=\left\{c_{1}, c_{2}, c_{3}, c_{4}, c_{5}, c_{6}, c_{7}\right\}$ 
and $C_{8}=\left\{c_{1}, c_{2}, c_{3}, c_{4}, c_{5}, c_{6}, c_{7}, c_{8}\right\}$ and binary operation . is defined on $C_{7}$ and $C_{8}$ in such way that $C_{7}$ and $C_{8}$ are LA-Semigroups and first four elements forms Klein-4 group; see "Extension-1 Table In [21].

TABLE 6. Extension-1 Table

\begin{tabular}{|c|c|c|c|c|c|c|c|c|c|c|c|c|c|c|c|c|c|}
\hline$\cdot$ & $c_{1}$ & $c_{2}$ & $c_{3}$ & $c_{4}$ & $c_{5}$ & $c_{6}$ & $c_{7}$ & &. & $c_{1}$ & $c_{2}$ & $c_{3}$ & $c_{4}$ & $c_{5}$ & $c_{6}$ & $c_{7}$ & $c_{8}$ \\
\hline$c_{1}$ & $c_{1}$ & $c_{2}$ & $c_{3}$ & $c_{4}$ & $c_{5}$ & $c_{5}$ & $c_{5}$ & & $c_{1}$ & $c_{1}$ & $c_{2}$ & $c_{3}$ & $c_{4}$ & $c_{5}$ & $c_{5}$ & $c_{5}$ & $c_{5}$ \\
\hline$c_{2}$ & $c_{2}$ & $c_{1}$ & $c_{4}$ & $c_{4}$ & $c_{5}$ & $c_{5}$ & $c_{5}$ & & $c_{2}$ & $c_{2}$ & $c_{1}$ & $c_{4}$ & $c_{4}$ & $c_{5}$ & $c_{5}$ & $c_{5}$ & $c_{5}$ \\
\hline$c_{3}$ & $c_{3}$ & $c_{4}$ & $c_{1}$ & $c_{2}$ & $c_{5}$ & $c_{5}$ & $c_{5}$ & $c_{3}$ & $c_{3}$ & $c_{4}$ & $c_{1}$ & $c_{2}$ & $c_{5}$ & $c_{5}$ & $c_{5}$ & $c_{5}$ \\
\hline$c_{4}$ & $c_{4}$ & $c_{3}$ & $c_{2}$ & $c_{1}$ & $c_{5}$ & $c_{5}$ & $c_{5}$ & $c_{4}$ & $c_{4}$ & $c_{3}$ & $c_{2}$ & $c_{1}$ & $c_{5}$ & $c_{5}$ & $c_{5}$ & $c_{5}$ \\
\hline$c_{5}$ & $c_{5}$ & $c_{5}$ & $c_{5}$ & $c_{5}$ & $c_{5}$ & $c_{5}$ & $c_{5}$ & $c_{5}$ & $c_{5}$ & $c_{5}$ & $c_{5}$ & $c_{5}$ & $c_{5}$ & $c_{5}$ & $c_{5}$ & $c_{5}$ \\
\hline$c_{6}$ & $c_{5}$ & $c_{5}$ & $c_{5}$ & $c_{5}$ & $c_{5}$ & $c_{5}$ & $c_{5}$ & $c_{6}$ & $c_{5}$ & $c_{5}$ & $c_{5}$ & $c_{5}$ & $c_{5}$ & $c_{5}$ & $c_{5}$ & $c_{5}$ \\
\hline$c_{7}$ & $c_{5}$ & $c_{5}$ & $c_{5}$ & $c_{5}$ & $c_{5}$ & $c_{6}$ & $c_{5}$ & $c_{7}$ & $c_{5}$ & $c_{5}$ & $c_{5}$ & $c_{5}$ & $c_{5}$ & $c_{6}$ & $c_{5}$ & $c_{5}$ \\
\hline & & & & & & & & & $c_{8}$ & $c_{5}$ & $c_{5}$ & $c_{5}$ & $c_{5}$ & $c_{5}$ & $c_{5}$ & $c_{5}$ & $c_{8}$ \\
\hline
\end{tabular}

Extension-2 in [21]: This idea can be extended to infinite set on which binary operation can be defined is in such way that the set having infinite idempotents in which only one element is zero element and the set containing all the elements that are idempotents forms commutative orthodox semigroup e.g. If we take set of non negative integers (say $\mathrm{W})$ set of whole numbers and binary operation is defined on $\mathrm{W}$ in such way that 0 becomes zero element in $\mathrm{W}$ and the subset of $\mathrm{W}$ say $W_{1}$ that includes elements 1, 2, 3 and 4 forms Klein-4 group. The set $W_{2}=\{8,10,12,14, \ldots\}$ is set of idempotents while the set $W_{3}=\{7,9,11,13, \ldots\}$ is set in which every element is self zero divisor and the product of each element in $W_{3}$ with each element of $\mathrm{W}$ gives 0 . Let $W_{4}=\{5,6\}$ and $5.5=6.6=6.5=0$ but $5.6=6$. Thus $\mathrm{W}$ w.r.t binary operation . is infinite locally associative LA-Semigroup with zero element 0 and this contains $W-1$ which Klein-4 group and the subset of $\mathrm{W}$ say $W_{A}=\{0,5,6\}$ is locally associative LA-Semigroup with zero element 0 . The subset of $\mathrm{W}$ say $W_{B}=\{0,8,10,12,14, \ldots\}$ is commutative orthodox semigroup and $W_{B}$ is also ideal of W. This is example in Case-3 in which first four elements do not form Klein-4 group.

Extension-3 in [21]: This is not difficult to construct a LA-Semigroup with zero element that contains cyclic group as well as Klein-4 group. 
TABle 7. Extension-3 $(A)$ and Extension-3 $(B)$ Table

\begin{tabular}{|c|c|c|c|c|c|c|c|c|c|c|c|c|c|c|c|c|c|c|c|c|c|c|c|}
\hline$*$ & 1 & 2 & 3 & 4 & 5 & 6 & 7 & 8 & 9 & 10 & & $\cdot$ & 1 & 2 & 3 & 4 & 5 & 6 & 7 & 8 & 9 & 10 & 11 \\
\hline 1 & 1 & 2 & 3 & 4 & 5 & 5 & 5 & 5 & 5 & 5 & & 1 & 1 & 2 & 3 & 4 & 5 & 5 & 5 & 5 & 5 & 5 & 5 \\
\hline 2 & 2 & 1 & 4 & 4 & 5 & 5 & 5 & 5 & 5 & 5 & & 2 & 2 & 1 & 4 & 4 & 5 & 5 & 5 & 5 & 5 & 5 & 5 \\
\hline 3 & 3 & 4 & 1 & 2 & 5 & 5 & 5 & 5 & 5 & 5 & & 3 & 3 & 4 & 1 & 2 & 5 & 5 & 5 & 5 & 5 & 5 & 5 \\
\hline 4 & 4 & 3 & 2 & 1 & 5 & 5 & 5 & 5 & 5 & 5 & & 4 & 4 & 3 & 2 & 1 & 5 & 5 & 5 & 5 & 5 & 5 & 5 \\
\hline 5 & 5 & 5 & 5 & 5 & 5 & 5 & 5 & 5 & 5 & 5 & & 5 & 5 & 5 & 5 & 5 & 5 & 5 & 5 & 5 & 5 & 5 & 5 \\
\hline 6 & 5 & 5 & 5 & 5 & 5 & 5 & 5 & 5 & 5 & 5 & & 6 & 5 & 5 & 5 & 5 & 5 & 6 & 5 & 5 & 5 & 5 & 5 \\
\hline 7 & 5 & 5 & 5 & 5 & 5 & 6 & 5 & 5 & 5 & 5 & & 7 & 5 & 5 & 5 & 5 & 5 & 5 & 5 & 8 & 5 & 5 & 5 \\
\hline 8 & 5 & 5 & 5 & 5 & 5 & 5 & 5 & 8 & 9 & 10 & & 8 & 5 & 5 & 5 & 5 & 5 & 5 & 5 & 5 & 5 & 5 & 5 \\
\hline 9 & 5 & 5 & 5 & 5 & 5 & 5 & 5 & 9 & 10 & 8 & & 9 & 5 & 5 & 5 & 5 & 5 & 5 & 5 & 5 & 5 & 5 & 5 \\
\hline 10 & 5 & 5 & 5 & 5 & 5 & 5 & 5 & 10 & 1 & 9 & & 10 & 5 & 5 & 5 & 5 & 5 & 5 & 5 & 5 & 5 & 5 & 5 \\
\hline & & & & & & & & & & & & 11 & 5 & 5 & 5 & 5 & 5 & 5 & 5 & 5 & 5 & 5 & 5 \\
\hline
\end{tabular}

Extension-4 in [21]: We construct LA-Semigroup containing cyclic group and commutative orthodox semigroup which is locally associative and contain zero element elaborated in "Extension-4 Table".

TABLE 8. Extension-4 Table

\begin{tabular}{|l|l|l|l|l|l|l|l|l|l|l|l|l|l|l|l|l|l|l|l|}
\hline$\cdot$ & 1 & 2 & 3 & 4 & 5 & 6 & 7 & 8 & &. & 1 & 2 & 3 & 4 & 5 & 6 & 7 & 8 & 9 \\
\hline 1 & 1 & 2 & 3 & 4 & 5 & 5 & 5 & 5 & & 1 & 1 & 2 & 3 & 4 & 5 & 5 & 5 & 5 & 5 \\
\hline 2 & 2 & 3 & 4 & 1 & 5 & 5 & 5 & 5 & & 2 & 2 & 3 & 4 & 1 & 5 & 5 & 5 & 5 & 5 \\
\hline 3 & 3 & 4 & 1 & 2 & 5 & 5 & 5 & 5 & & 3 & 3 & 4 & 1 & 2 & 5 & 5 & 5 & 5 & 5 \\
\hline 4 & 4 & 1 & 2 & 3 & 5 & 5 & 5 & 5 & & 4 & 4 & 1 & 2 & 3 & 5 & 5 & 5 & 5 & 5 \\
\hline 5 & 5 & 5 & 5 & 5 & 5 & 5 & 5 & 5 & & 5 & 5 & 5 & 5 & 5 & 5 & 5 & 5 & 5 & 5 \\
\hline 6 & 5 & 5 & 5 & 5 & 5 & 5 & 5 & 5 & & 5 & 5 & 5 & 5 & 5 & 5 & 5 & 5 & 5 & 5 \\
\hline 7 & 5 & 5 & 5 & 5 & 5 & 6 & 5 & 5 & & 5 & 5 & 5 & 5 & 5 & 5 & 5 & 5 & 5 & 5 \\
\hline 8 & 5 & 5 & 5 & 5 & 5 & 5 & 5 & 8 & & 5 & 5 & 5 & 5 & 5 & 5 & 5 & 5 & 5 & 5 \\
\hline & & & & & & & & & & 5 & 5 & 5 & 5 & 5 & 5 & 5 & 5 & 5 & 5 \\
\hline
\end{tabular}

Extension-5 Towards LA-Semigroups with Left Zero Element: We constructed LASemigroups which is not locally associative and contains left zero element on sets $S_{3}$ and $S_{4}$. We use acronym LZLA-Semigroup. Following is table for Tables for $S_{3}$ and $S_{4}$ :

TABLE 9. Extension-5 Table

\begin{tabular}{|c|c|c|c|c|c|c|c|c|c|c|c|c|c|c|c|}
\hline$\cdot$ & 1 & 2 & 3 & & $\cdot$ & 1 & 2 & 3 & 4 & & $\eta$ & 1 & 2 & 3 & 4 \\
\hline 1 & 1 & 1 & 1 & & 1 & 1 & 1 & 1 & 1 & & 1 & 1 & 1 & 1 & 1 \\
\hline 2 & 3 & 1 & 1 & & 2 & 1 & 1 & 1 & 1 & & 2 & 1 & 1 & 1 & 1 \\
\hline 3 & 1 & 1 & 1 & & 3 & 2 & 1 & 1 & 1 & & 3 & 1 & 1 & 1 & 1 \\
\hline & & & & & 4 & 1 & 1 & 1 & 1 & & 4 & 2 & 1 & 1 & 1 \\
\hline & & & & & & & & & & & & & & & \\
\hline$\eta$ & 1 & 2 & 3 & & $*$ & 1 & 2 & 3 & 4 & & $\theta$ & 1 & 2 & 3 & 4 \\
\hline 1 & 1 & 1 & 1 & & 1 & 1 & 1 & 1 & 1 & & 1 & 1 & 1 & 1 & 1 \\
\hline 2 & 1 & 1 & 1 & & 2 & 1 & 1 & 1 & 1 & & 2 & 1 & 1 & 1 & 1 \\
\hline 3 & 2 & 1 & 1 & & 3 & 4 & 1 & 1 & 1 & & 3 & 1 & 1 & 1 & 1 \\
\hline & & & & & 4 & 1 & 1 & 1 & 1 & & 4 & 3 & 1 & 1 & 1 \\
\hline
\end{tabular}

\section{Remarks on 5th Extension}

(a) This is LA-Semigroup with left zero element in which product of each element with 
itself is left zero element.

(b) This is not locally associative LA-Semigroup.

(c) Each ideal of LZLA-Semigroups in Table For LZLA-Semigroup is right ideal and right ideal I does not contain element $\mathrm{b}$ such that $b . L=c$ where $b \neq c$ and $\mathrm{L}$ means left zero element of LA-Semigroup.

(d) If any semigroup $\mathrm{S}$ or LA-Semigroup $\mathrm{T}$ contains left zero element then the subset of $\mathrm{S}$ or $\mathrm{T}$ that contains left zero element is right ideal of $\mathrm{S}$ or $\mathrm{T}$.

Extension-6 Towards RA-Semigroup with Right Zero Element: We extend the idea of LA-Semigroups and constructed RA-Semigroups with right zero element by the following table:

TABLE 10. Extension-6 Table

\begin{tabular}{|c|c|c|c|c|c|c|c|c|c|c|c|c|c|c|c|}
\hline$\cdot$ & 1 & 2 & 3 & & $\cdot$ & 1 & 2 & 3 & 4 & & $\eta$ & 1 & 2 & 3 & 4 \\
\hline 1 & 1 & 3 & 1 & & 1 & 1 & 3 & 1 & 1 & & 1 & 1 & 1 & 2 & 1 \\
\hline 2 & 1 & 1 & 1 & & 2 & 1 & 1 & 1 & 1 & & 2 & 1 & 1 & 1 & 1 \\
\hline 3 & 1 & 1 & 1 & & 3 & 1 & 1 & 1 & 1 & & 3 & 1 & 1 & 1 & 1 \\
\hline & & & & & 4 & 1 & 1 & 1 & 1 & & 4 & 1 & 1 & 1 & 1 \\
\hline & & & & & & & & & & & & & & & \\
\hline$\eta$ & 1 & 2 & 3 & & $*$ & 1 & 2 & 3 & 4 & & $\theta$ & 1 & 2 & 3 & 4 \\
\hline 1 & 1 & 1 & 2 & & 1 & 1 & 4 & 1 & 1 & & 1 & 1 & 1 & 4 & 1 \\
\hline 2 & 1 & 1 & 1 & & 2 & 1 & 1 & 1 & 1 & & 2 & 1 & 1 & 1 & 1 \\
\hline 3 & 1 & 1 & 1 & & 3 & 1 & 1 & 1 & 1 & & 3 & 1 & 1 & 1 & 1 \\
\hline & & & & & 4 & 1 & 1 & 1 & 1 & & 4 & 1 & 1 & 1 & 1 \\
\hline
\end{tabular}

\section{Remarks on 6th Extension}

(a) This is RA-Semigroup with right zero element in which product of each element with itself is right zero.

(b) This is not locally associative RA-Semigroup.

(c) Each ideal of RZRA-Semigroups in Table For RZRA-Semigroup is left ideal and left ideal I does not contain element $\mathrm{b}$ such that $b . M=c$ where $b \neq c$ and $\mathrm{M}$ means right zero element of RA-Semigroup.

(d) If any semigroup $\mathrm{S}$ or RA-Semigroup $\mathrm{T}$ contains right zero element then the subset of $\mathrm{S}$ or $\mathrm{T}$ that contains right zero element is left ideal of $\mathrm{S}$ or $\mathrm{T}$.

\section{Extensions towards LA-Semigroup and RA-Semigroup without Zero Element}

We constructed examples on LA-Semigroups with left zero element and RA-Semigroups with right zero element by the following tables:

TABLE 11. Table for LA-Semigroup and RA-Semigroup without Zero Element

\begin{tabular}{|c|c|c|c|c|c|c|c|c|}
\hline$*$ & $\mathrm{x}$ & $\mathrm{y}$ & $\mathrm{z}$ & & $\cdot$ & $\mathrm{x}$ & $\mathrm{y}$ & $\mathrm{z}$ \\
\hline $\mathrm{x}$ & $\mathrm{y}$ & $\mathrm{z}$ & $\mathrm{y}$ & & $\mathrm{x}$ & $\mathrm{y}$ & $\mathrm{z}$ & $\mathrm{z}$ \\
\hline $\mathrm{y}$ & $\mathrm{z}$ & $\mathrm{z}$ & $\mathrm{z}$ & & $\mathrm{y}$ & $\mathrm{z}$ & $\mathrm{z}$ & $\mathrm{z}$ \\
\hline $\mathrm{z}$ & $\mathrm{z}$ & $\mathrm{z}$ & $\mathrm{z}$ & & $\mathrm{z}$ & $\mathrm{y}$ & $\mathrm{z}$ & $\mathrm{z}$ \\
\hline
\end{tabular}




\subsection{Indicating Error in Paramedial Groupoid.}

Sawatraksa and Namnak constructed an example on a set $\bar{A}$ and discussed that this semigroup holds paramedial law by the following table:

TABLE 12. Table $T-1$ By Sawatraksa and Namnak

\begin{tabular}{|c|c|c|c|}
. & $\mathrm{a}$ & $\mathrm{b}$ & $\mathrm{c}$ \\
\hline $\mathrm{a}$ & $\mathrm{a}$ & $\mathrm{a}$ & $\mathrm{a}$ \\
\hline $\mathrm{b}$ & $\mathrm{a}$ & $\mathrm{a}$ & $\mathrm{a}$ \\
\hline $\mathrm{c}$ & $\mathrm{a}$ & $\mathrm{b}$ & $\mathrm{c}$ \\
\hline
\end{tabular}

Clearly from table $\bar{A}$ is semigroup with left identity but $\bar{A}$ is not paramedial if we see $(b c)(c c)=a c=a$ but $(c c)(c b)=c b=b$ i.e. the condition $(a b)(c d)=(d b)(c a)$ is not satisfied.

This is an example of three square matrices of order $2 \times 2$, by letting $M_{1}=\left(\begin{array}{ll}0 & 0 \\ 0 & 0\end{array}\right)$, $M_{2}=\left(\begin{array}{ll}0 & 1 \\ 0 & 0\end{array}\right)$ and $M_{2}=\left(\begin{array}{ll}1 & 0 \\ 0 & 0\end{array}\right)$ respectively. Clearly The set say $\bar{M}=\left\{M_{1}, M_{2}, M_{3}\right\}$ is closed, associative and semigroup with left identity w.r.t matrix multiplication but $S$ does not satisfy paramedial law.

If we take set say $\bar{N}=\left\{N_{1}, N_{2}, N_{3}\right\}$ where $N_{1}=\left(\begin{array}{ll}0 & 0 \\ 0 & 0\end{array}\right), N_{2}=\left(\begin{array}{ll}0 & 1 \\ 0 & 0\end{array}\right)$ and $N_{3}=\left(\begin{array}{ll}0 & 0 \\ 0 & 1\end{array}\right)$ then $\bar{N}$ w.r.t matrix multiplication is semigroup with right identity but does not hold paramedial law.

So the claim of Sawatraksa and Namnak in Example 2.2 [18] is not correct. Also we can extend this idea of semigroup with left identity and semigroup with right identity on infinite set either countable or uncountable e.g. if we take set $M_{1}$ that contains matrices of order $2 \times 2$ having sets $O=\left(\begin{array}{ll}0 & 0 \\ 0 & 0\end{array}\right), B_{N}=\left(\begin{array}{ll}0 & n \\ 0 & 0\end{array}\right)$ and $A=\left(\begin{array}{ll}1 & 0 \\ 0 & 0\end{array}\right)$ where $B_{N}$ means all square matrices of order $2 \times 2$ in which $n \neq 0$ and $\mathrm{n}$ is any natural number then the set $M_{1}$ is semigroup with left identity and by the same way if we have set $B_{R}$ where instead of $n$ if we use $r$ where $r$ is any real number other than zero then we have example of semigroup with left identity on uncountable set.

Similarly if we have set $N_{1}$ having square matrices of order $2 \times 2$ which are $O=\left(\begin{array}{ll}0 & 0 \\ 0 & 0\end{array}\right)$, $B_{N}=\left(\begin{array}{cc}0 & \mathrm{n} \\ 0 & 0\end{array}\right)$ and $C=\left(\begin{array}{ll}0 & 0 \\ 0 & 1\end{array}\right)$ where $B_{N}$ means all square matrices of order $2 \times 2$ in which $n \neq 0$ and $\mathrm{n}$ is any natural number then the set $N_{1}$ is semigroup with right identity 
and by the same way if we have set $B_{R}$ where instead of $\mathrm{n}$ if we use $\mathrm{r}$ where $\mathrm{r}$ is any real number other than zero then we have example of semigroup with right identity on uncountable set.

Note: In above examples semigroups hold medial law. Semigroup with left identity is medial and LDD-Semigroup while semigroup with right identity is medial and RDDSemigroup. Further we have following example on non commutative semigroup with two left(right) identities holding LDD(RDD)-law:

Example of Semigroup, medial and LDD-Semigroup with Two Left Identities: If we define binary operation $\mu$ on $R$ by $a \mu b=|a| . b$ then $R$ is semigroup, medial and LDD-Semigroup with two left identities -1 and 1.

Example of Semigroup, medial and RDD-Semigroup with Two Right Identities: If we define binary operation $\nu$ on $R$ by $a \nu b=a .|b|$ then $R$ is semigroup, medial and RDD-Semigroup with two right identities -1 and 1 .

\section{Remark 3.24.}

(i) If $D$ with left identity holds medial law then $D$ also holds $L D D$-law and vice versa.

(ii) If $D$ with right identity holds medial law then $D$ also holds $R D D$-law and vice versa.

We further investigated that some non commutative idempotent semigroups i.e. bands holds either medial and LDD-law or medial as well as RDD-law explained by the following examples:

\section{Examples of Non Commutative Bands which are Medial and LDD-Semigroup:}

(a): The set of all constant functions from set $S$ to $S$ w.r.t binary operation composition of mapping.

(b): The set of all collinear vectors say $\bar{V}$ w.r.t binary operation $u v=(\hat{u} \cdot v) \cdot \hat{v} \forall u$ and $v \in$ $\bar{V}$ where $\hat{u}$ and $\hat{v}$ are unit vectors of vectors $u$ and $v$ respectively.

(c): The power set of non empty set $S$ say $P(S)$ w.r.t binary operation defined by $A B=$ $(A \cap B)-B^{c}$.

Examples of Non Commutative Bands which are Medial and RDD-Semigroup:

(a): The set of all constant functions from set $S$ to $S$ w.r.t binary operation composition of functions.

(b): The set of all collinear vectors say $\bar{V}$ w.r.t binary operation $u v=(\hat{v} \cdot u) \cdot \hat{u} \forall u, v \in \bar{V}$ where $\hat{u}$ and $\hat{v}$ are unit vectors of vectors $u$ and $v$ respectively.

(c:) The power set of non empty set $S$ say $P(S)$ w.r.t binary operation defined by $A B=$ $(A \cap B)-A^{c}$.

3.25. Results on n-dimensional LA(RA)-Semigroups, Non Commuttaive as well as Commutative Medials.

In this section we developed some results on finite as well as infinite LA-Semigroups, 
RA-Semigroups, n-dimensional LA-Semigroup, n-dimensional RA-Semigroup, non commutative medials with more than two left or right identities and commutative idempotent medial groupoids.

Through out this section we will use $\mathrm{f}$ and $\mathrm{g}$ which are defined functions from $R$ to $R, Q$ to $Q, Z$ to $Z$ and $Z_{n}$ to $Z_{n}$. For readers to grasp the idea of results, we constructed following examples:

\section{Example 3.25.1: Binary Operation to Construct Infinite LA-Semigroup}

If we define binary operation $*$ on $R$ or $Q$ by $a * b=f(a)+f^{2}(b)$ then $R$ is LA-Semigroup w.r.t the following conditions:

(a) $f(x)=0$ then $R$ or $Q$ are zero semigroups with zero element 0 .

(b) $f(x)=k$ where $\mathrm{k}$ is any constant number then again $R$ and $Q$ are zero semigroups with zero element $2 . k$.

(c) $f(x)=x$ i.e. identity function then $R$ or $Q$ are commutative groups because this simply means that $a b=a+b$.

(d) $f(x)=-x$ i.e. negative identity function then $R$ or $Q$ are LA-Groups with left identity element 0 and each element is self inverse.

Note in 3.25.1: Here $f^{2}(x)$ means $f(f(x))$ i.e. composition of functions or composite function rule.

\section{Example 3.25.2: Binary Operation to Construct Infinite RA-Semigroup}

If we define binary operation $\eta$ on $R$ by $a \eta b=f^{2}(a)+f(b)$ then $R$ is RA-Semigroup w.r.t following conditions:

(a) $f(x)=0$ then $R$ is zero semigroup with zero element 0 .

(b) $f(x)=k$ where $\mathrm{k}$ is any constant number then again $R$ is zero semigroup with zero element $2 . k$.

(c) $f(x)=x$ i.e. identity function then $R$ is commutative group because this simply means that $a b=a+b$.

(d) $f(x)=-x$ i.e. negative identity function then $R$ is RA-Group with right identity element 0 and each element is self inverse.

Note in 3.25.2: Here $f^{2}(x)$ means $f(f(x))$ i.e. composition of functions or composite function rule.

\section{Example 3.25.3: Binary Operation to Construct Different Algebraic Structures}

If we define binary operation $\kappa$ on $R$ by $a \kappa b=f(a)+g(b)$ then following conditions holds:

(a) If $f(x)=g(x)=0$ then this is case of zero semigroup.

(b) If $f(x)=g(x)=k$ then this is case of zero semigroup with zero element $\mathrm{k}$.

(c) If $f(x)=g(x)=x$ then this is case of cyclic group.

(d) If $f(x)=g(x)=-x$ then this is case of non associative but commutative groupoid that satisfies medial as well paramedial law.

(e) If $f(x)=x$ and $g(x)=-x$ then this is case of LA-Semigroup.

(f) If $f(x)=-x$ and $g(x)=x$ then this is case of RA-Semigroup. 
(g) If $f(x)=x$ and $g(x)=0$ then this is case of semigroup which is medial as well as RDD-semigroup.

(h) If $f(x)=0$ and $g(x)=x$ then this is case of semigroup which is medial as well as LDD-semigroup.

(i) If $f(x)=-x$ and $g(x)=0$ then this is case of medial and RDD-semigroup.

(j) If $f(x)=0$ and $g(x)=-x$ then this is case of medial and LDD-semigroup.

\section{Example 3.25.4: Binary Operation to Construct n-dimensional LA-Semigroup and} RA-Semigroup

(a): If we define binary operation on $R^{3}$ by $x \sigma y=(a, b, c) \sigma(d, e, f)=(c+d, b+e, a+$ $f$ ) then $R^{3}$ holds left invertive law.

(b): If we define binary operation on $R^{3}$ by $x \sigma y=(a, b, c) \sigma(d, e, f)=(a+f, b+$ $e, c+d)$ then $R^{3}$ holds right invertive law.

(c): If we define binary operation on $R^{n}$ by $x \sigma y=\left(x_{1}, x_{2}, x_{3}, \ldots, x_{n}\right) \sigma\left(y_{1}, y_{2}, y_{3}, \ldots, y_{n}\right)=$ $\left(x_{n}+y_{1}, x_{n-1}+y_{2}, x_{n-2}+y_{3}, \ldots, x_{1}+y_{n}\right)$ then $R^{n}$ is LA-Semigroup.

(d): If we define binary operation on $R^{n}$ by $x \sigma y=\left(x_{1}, x_{2}, x_{3}, \ldots, x_{n}\right) \varsigma\left(y_{1}, y_{2}, y_{3}, \ldots, y_{n}\right)=$ $\left(x_{1}+y_{n}, x_{2}+y_{n-1}, x_{3}+y_{n-2}, \ldots, x_{n}+y_{1}\right)$ then $R^{n}$ is RA-Semigroup.

Note in 3.25.4: The same procedure can be done for multiplication and by this way we can develop the concept of LA-Ring, RA-Ring, LA-Field and RA-Field.

\section{Example 3.25.5: Binary Operation to Construct Non Commutative Medials with more than Two Left(Right) Identities}

If $\mathrm{n}$ is finite positive even number with the condition $n \geq 6$ and we define binary operation on $Z_{n}$ by $x y=[y+k x]_{\bmod n}$ where $k=\frac{n}{2}$ then $Z_{n}$ is finite non commutative medial with $k$ idempotents and these $k$ idempotents are also left identities. The subset of $Z_{n}$ say $Z_{L}$ containing elements $\{0,2,4, \ldots, k-2, k, k+2, k+4, \ldots, n-2\}$ forms non commutative band that holds medial as well as LDD-law.

$Z_{n}$ is medial and contains $\mathrm{k}$ idempotents which are right identities if $\mathrm{n}$ is finite positive even number with the condition $n \geq 6$ and we define binary operation on $Z_{n}$ by $x y=[x+k y]_{\bmod n}$ where $k=\frac{n}{2}$.

In this case the subset of $Z_{n}$ say $Z_{R}$ containing elements $\{0,2,4, \ldots, k-2, k, k+2, k+$ $4, \ldots, n-2\}$ forms non commutative band that holds medial as well as RDD-law.

Note in 3.25.5: $Z_{n}$ also holds medial law with three or more than three left or right identities if $\mathrm{n}$ is finite positive odd composite number or even number and we define following binary operations:

$$
\begin{array}{ll}
x y=(y+6 x)_{\bmod 48} & x y=(y+5 x)_{\bmod 5} \\
x y=(x+6 y)_{\bmod 48} & x y=(x+5 y)_{\bmod 35}
\end{array}
$$

\section{Example 3.25.6: Binary Operation to Construct Commutative Medial}


(i) If $n \geq 3$ and we define binary operation $\tau$ on $Z_{n}$ by $x \tau y=[f(x)+f(y)]_{\bmod n}$ then $Z_{n}$ is commutative medial w.r.t following conditions:

(a) If $f(x)=0$ is defined on $Z_{n}$ then $Z_{n}$ is zero semigroup with zero element 0 .

(b) If $f(x)=x$ i.e. identity function then $Z_{n}$ is cyclic group.

(c) If $f(x)=-x$ i.e. negative identity function then $Z_{n}$ is cyclic group if $n=2$, and if $n>2$ then $Z_{n}$ is commutative groupoid that satisfies medial law but does not satisfy associative law. If $\mathrm{n}$ is finite positive even number with condition $n \geq 4$ then the subset of $Z_{n}$ say $Z_{C}=\left\{0, \frac{n}{2}\right\}$ is cyclic group.

Note in (a): $Z_{n}$ is not medial if we define $f(x)=x^{k}$ where $k \in\{0,1,2, \ldots, n-$ $1\}-\{0,1\}$ e.g. if we define binary operation on $Z_{8}$ by $x y=x^{2}+y^{2}$.

Also if we define binary operation $x y=(x+y)^{2}$ on $Z_{8}$ then surely $Z_{8}$ is neither associative nor medial groupoid but $Z_{8}$ is commutative.

(ii): If we define binary operation on $R$ or $Q$ by $u \kappa v=\frac{(u+v)}{k}$ where $\mathrm{k}$ is any real number other than 0 and 1 then $R$ or $Q$ is commutative medial and if $k=2$ then $R$ or $Q$ are commutative idempotent medial.

(iii): If we define binary operation on $Z_{n}$ by $x \kappa y=\left[\left(\frac{n+1}{2}\right)(x+y)\right]_{\bmod n}$ where $\mathrm{n}$ is finite positive odd number with the condition $n \geq 3$ then $Z_{n}$ is finite commutative idempotent medial.

\section{Example 3.25.7: Binary Operation to Construct Medials}

If we define binary operations ${ }_{*}, ., \theta, \eta, \kappa, \mu, \nu, \rho$ and $\varrho$ on $Z_{10}$ by the following ways:

$a_{*} b=(a+b+k)_{\bmod 10}$

$a . b=(a+2 . b+2 . k)_{\bmod 10}$

$a \theta b=(a+3 . b+3 . k)_{\bmod 10}$

$a \eta b=(a+4 . b+4 . k)_{\bmod 10}$

$a \kappa b=(a+5 . b+5 . k)_{\bmod 10}$

$a \mu b=(a+6 . b+6 . k)_{\bmod 10}$

$a \nu b=(a+7 . b+7 . k)_{\bmod 10}$

$a \rho b=(a+8 . b+8 . k)_{\bmod 10}$

$a \varrho b=(a+9 . b+9 . k)_{\bmod 10}$

Here we fix $k=1$ and have following results:

With respect to $*, ., \theta$ and $\nu$ the subset $\{9,4\}$ forms cyclic group with identity 9 .

With respect to $\eta, \mu$ and $\rho\{4,9\}$ forms semigroup which also satisfies medial law as well as RDD-law.

With respect to $\kappa$ the subset $\{1,3,5,7,9\}$ forms semigroup which also satisfies medial law as well as RDD-law.

With respect to $\varrho Z_{10}$ is RA-Group with right identity 9 .

Note in Example 3.25.7:

(a) We use binary operation $a b=(a+L . b+L . k)_{\bmod n}$, and if $L=0$ then $a b=a$ which is case of semigroup, medial and RDD-Semigroup, and if $L=n-1$ with condition $k=0$ then $Z_{n}$ is RA-Group. 
(b) The same results can be converted into LA-Groups and LDD-Semigroups e.g. if we use binary operation $a b=(b+L . a+L . k)_{\bmod n}$, , and if $L=0$ then $a b=b$ which is case of semigroup, medial and LDD-Semigroup.

\section{CONCLUSION}

We concluded our results, simmilarities and dissimilarities of LA-Semigroup and RASemigroup with semigroup, and simmilarities and dissimilarities of LA-Group and RAGroup with group by the following remarks:

(i) Groups in which each element is self inverse are always commutative but this may not be true in case of LA-Groups and RA-Groups.

(ii) Both Medial and LA-Semigroup with left identity hold the condition $a(b c)=b(a c)$ but if medial $S$ contains left identity then $S$ may not be LA-Monoid e.g. if we define binary operation on $R$ by $a b=|a| . a^{k} . b$ where . means product and $\mathrm{k}$ is finite positive integer then $R$ is medial with one left identity 1 if $\mathrm{k}$ is finite positive odd number and $R$ is medial with two left identities -1 and 1 if $\mathrm{k}$ is finite positive even number.

(iii) Both Medial and RA-Semigroup with right identity hold the condition $(a b) c=(a c) b$ but if medial $S$ contains right identity then $S$ may not be RA-Monoid e.g. if we define binary operation on $R$ by $a b=a .|b| . b^{k}$ where . means product and $\mathrm{k}$ is finite positive integer then $R$ is medial with one right identity 1 if $\mathrm{k}$ is finite positive odd number and $R$ is medial with two right identities -1 and 1 if $\mathrm{k}$ is finite positive even number.

(iv) If any group $H$ or any LA-Semigroup $S$ satisfies the condition $(a b) c=(b a) c$ then $H$ and $S$ are commutative but if medial $U$ satisfy this property then $U$ is LDD-semigroup but may not be commutative. Similarly if any group $G$ or any RA-Semigroup $T$ satisfies the condition $a(b c)=a(c b)$ then $G$ and $T$ are commutative but if medial $V$ satisfy this property then $V$ is RDD-semigroup but may not be commutative.

(v) Non commutative group and non commutative monoid can not hold medial, paramedial, LDD, RDD, left invertive or right invertive law and further the relation of semigroup with paramedial is only commutative property.

(vi) Non commutative semigroup may be medial, LDD-Semigroup or RDD-Semigroup.

(vii) Every LA-Monoid as well as RA-Monoid holds paramedial law, and by converse paramedial with left(right) identity is LA(RA)-Monoid.

(viii) Non commutative semigroup may not hold medial law, LDD-law or RDD-law e.g. Free semigroup.

(ix) To construct neither commutative nor associative finite groupoid which is either LASemigroup or RA-Semigroup, the order of groupoid must be atleast 3 but groups, commutative bands and semigroups can be constructed of order 2 . Further every groupoid of order 1 is always commutative monoid, and may be group if we take singleton set $\{0\}$ and use binary operation of addition.

See "Tables For Groups and Semigroups of Order 2" in which binary operations for groups, commutative bands and semigroups of order 2 are defined by the following:

$a_{*} b=(a+b)_{\bmod 2}$

$a . b=(a \times b)$ with elements $\{-1,1\}$. 
$a \mu b=(a \times b)_{\bmod 2}$ with elements $\{0,1\}$.

$a \nu b=(a \times b)_{\bmod 6}$ with elements $\{1,3\}$.

$a \eta b=(a . b)_{\bmod 4}$ where we take subset of $Z_{4}$ say $2 Z_{4}=\{0,2\}$ in which 0 is zero element. $a \rho b=(a . b)_{\bmod 24}$ where we take elements $\{4,16\}$ in which 16 is zero element.

$a \theta b=a$ for constant functions from $A$ to $A$ where $A=\{1,2\}$ and binary operation is composition of functions or composite function rule.

$a \kappa b=b$ for constant functions from $A$ to $A$ where $A=\{1,2\}$ and binary operation is composition of mapping.

TABLE 13. Table For Groups and Semigroups of Order 2

\begin{tabular}{|c|c|c|c|c|c|c|c|c|c|c|c|c|c|c|}
\hline$*$ & $\mathrm{a}$ & $\mathrm{b}$ & &. & $\mathrm{a}$ & $\mathrm{b}$ & & $\mu$ & $\mathrm{a}$ & $\mathrm{b}$ & & $\nu$ & $\mathrm{c}$ & $\mathrm{d}$ \\
\hline $\mathrm{a}$ & $\mathrm{a}$ & $\mathrm{b}$ & & $\mathrm{a}$ & $\mathrm{b}$ & $\mathrm{a}$ & & $\mathrm{a}$ & $\mathrm{a}$ & $\mathrm{b}$ & & $\mathrm{a}$ & $\mathrm{a}$ & $\mathrm{a}$ \\
\hline $\mathrm{b}$ & $\mathrm{b}$ & $\mathrm{a}$ & & $\mathrm{b}$ & $\mathrm{a}$ & $\mathrm{b}$ & & $\mathrm{b}$ & $\mathrm{b}$ & $\mathrm{b}$ & & $\mathrm{b}$ & $\mathrm{a}$ & $\mathrm{b}$ \\
\hline & & & & & & & & & & & & & & \\
\hline$\rho$ & $\mathrm{a}$ & $\mathrm{b}$ & & $\eta$ & $\mathrm{a}$ & $\mathrm{b}$ & & $\theta$ & $\mathrm{a}$ & $\mathrm{b}$ & & $\kappa$ & $\mathrm{a}$ & $\mathrm{b}$ \\
\hline $\mathrm{a}$ & $\mathrm{a}$ & $\mathrm{a}$ & & $\mathrm{a}$ & $\mathrm{b}$ & $\mathrm{b}$ & & $\mathrm{a}$ & $\mathrm{a}$ & $\mathrm{a}$ & & $\mathrm{a}$ & $\mathrm{a}$ & $\mathrm{b}$ \\
\hline $\mathrm{b}$ & $\mathrm{a}$ & $\mathrm{a}$ & & $\mathrm{b}$ & $\mathrm{b}$ & $\mathrm{b}$ & & $\mathrm{b}$ & $\mathrm{b}$ & $\mathrm{b}$ & & $\mathrm{b}$ & $\mathrm{a}$ & $\mathrm{b}$ \\
\hline
\end{tabular}

(x) Finite semigroups always contain atleast one idempotent [22, 5] but finite LA-Semigroup, RA-Semigroup, medial, medial and either LDD-Semigroup or RDD-Semigroup may not contain any idempotent.

(xi) The smallest medial and LDD-Semigroup has order 2 e.g. if we define binary operation $a b=(b+1)_{\bmod 2}$ on $Z_{2}$ and by the same way the smallest medial and RDD-Semigroup has also order 2 e.g. if we define binary operation $a b=(a+1)_{\bmod 2}$ on $Z_{2}$.

(xii) Near-Ring may be or may not be Ring but LA-Near Ring and LA-Ring are same terms, and by the same way RA-Near Ring and RA-ring are same terms.

(xiii) If semigroup $D$ with left identity holds LDD-law then $S$ also holds medial law and vice versa and by the same way if semigroup $T$ with right identity holds RDD-law then $S$ also holds medial law and vice versa.

\section{REFERENCES}

[1] I. Ahmad, S. Rehman, M. Iqbal, Amanullah, A Note on Left Abelian Distributive LA-Semigroups, Punjab Uni.j. math, 52, No. 1 (2020) 47-63.

[2] N. Ahmad, M. Ali, F. Ali, A.M. Khattak, Left Double Displacement Semigroup: A First Result, Matriks Sains Matematik (MSMK), 2, No. 2 (2018) 37-39.

[3] A.H. Clifford, G.B. Preston, The Algebraic Theory of Semigroups, Amer. Math. Soc, Mathematical Surveys 7 Providence (1961 and 1967).

[4] J.R. Cho, J. Jezek, T. Kepka, Paramedial Groupoids, Czechoslovak Mathematical Journal, 49, No. 124 (1999) 277-290.

[5] A. Distler, Classification and Enumeration of Finite Semigroups, Ph.D Thesis, University of St. Andrews, (2010).

[6] J.M. Howie, An Introduction to Semigroup Theory, Academic Press (1976).

[7] J. Jazek, T. Kepka, Modular groupoids, Czechoslovak Mathematical Journal, 34, No. 3 (1984) 477-487.

[8] M. Kazim, M. Naseeruddin, On Almost Semigroups, Alig. Bull. Math., 2 (1971) 1-7.

[9] N. Kehayopulu, On Completely Regular Ordered Semigroups, Scinetiae Mathematicae, 1 (1998) 27-32. 
[10] M. Khan, F. Smarandache, S. Anis, Theory of Abel Grassmanns Groupoids, Educational Publishers Columbus, (2015).

[11] M. Khan, Some Studies In AG*-Groupoids, prr.hec.gov.pk (2008).

[12] N. Kimura, Note On Idempotent Semigroups, Tokyo Institute of Technology and Tulane University of Louisiana, 33 (1957) 642-645.

[13] N. Kimura, M. Yamada, Note On Idempotent Semigroups II, Proc. Japan Acad, 34 (1958) 110-112.

[14] Q. Mushtaq, S.M. Yusuf, On LA-Semigroups, Alig. Bull. Math., 8 (1978) 65-70.

[15] Q. Mushtaq, Abelian Groups Defined by LA-Semigroups, Studia Scient. Math.Hungar, 18 (1983) 427-428.

[16] Q. Mushtaq, M.S. Kamran, Finite AG-Groupoid with Left Identity and Left Zero, IJMMS, 27, No. 6 (2001) 387-389.

[17] N. Rehman, N. Shah, M.I. Ali, A. Ali, Generalized Roughness in $\left(\epsilon, \varepsilon V_{q}\right)$-Fuzzy Substructures of LASemigroup, J.Natn.Sci.Foundation Sri Lanka, 46, No. 3 (2018) 465-473.

[18] N. Sawatraksa, C. Namnak, Some Remarks on Paramedial Semigroups, Thai Journal of Mathematics (Special Issue), Annual Meeting in Mathematics, (2019) 167-176.

[19] D.A. Seguier, Elements De La Theorie Des Groupes Abstraits (Elements Of The Theory Of Abstract Groups) (1094).

[20] M. Shah, T. Shah, A. Ali, On the Cancellativity of AG-Groupoids, International Mathematical Forum, 6 (2011) 2187-2194.

[21] M. Shah, I. Ahmad, A. Ali, Discovery of New Classes of AG-Groupoids, Research Journal of Recent Sciences, 1, No. 11 (2012) 47-49.

[22] T.A. Whitelaw, An Introduction to Abstract Algebra, CRC Press (1st Edition), (1978) 64-68, Powers of an Element in Semigroup. 